\title{
Very High Energy Emission from Pulsars
}

\author{
Tadashi Kifune \\ Institute for Cosmic Ray Research, University of Tokyo
}

\begin{abstract}
Observations of gamma rays around $10^{12} \mathrm{eV}(=1 \mathrm{TeV}$ ) from pulsars are reviewed and the results are discussed and compared with emission at other wavelengths. Very high energy gamma rays do not appear to be modulated with the pulsar period, which suggests that a major part of the VHE emission is not from the pulsar magnetosphere.
\end{abstract}

\section{Introduction}

Observations of Very High Energy (VHE) gamma rays beyond $100 \mathrm{GeV}$ are made using the ground-based air Cerenkov technique. The large collecting area this technique provides is necessary for detecting the small photon flux, which rapidly decreases with energy following a non-thermal power law spectrum. Recently, the detection sensitivity at VHE energies was improved, and also the angular resolution narrowed to about $0.1^{\circ}$, with the development of the imaging Cerenkov technique (e.g. Cawley and Weekes 1995). The pioneering success of the technique preceded by several years the launch of the Compton Gamma Ray Observatory (CGRO) in 1991. The EGRET detections of GeV gamma rays (e.g. Ramanamurthy et al. 1995, and references therein), have resulted in pulsars becoming one of the most important targets of VHE observations, diverting from 1980 's attempts devoted mainly to X-ray and millisecond pulsars.

The detection of VHE gamma rays provides evidence of progenitor electrons at higher energies, accelerated by the induced electric field arising from the rapid rotation of the high magnetic field of the pulsar. A possible mechanism for radiating the VHE gamma rays is the inverse Compton process by which ambient soft photons at radio to optical wavelengths are scattered by the ( $>\mathrm{TeV}$ ) electrons and boosted to $\sim \mathrm{TeV}$ energies. It is, however, not clear under what conditions the presence of the energetic electrons can lead to VHE gamma ray emission; the alternative process of synchrotron radiation dissipates the energy of the progenitor electrons into softer photons. Furthermore, after production, energetic gamma rays can be absorbed within the source by conversion into an electron-positron pair. The various mechanisms have been discussed by a number of authors, and efforts made to model the complex cascade phenomena in the plasma of high energy electrons and positrons, and discern the role of the magnetic field structure near or at the pulsar (e.g. see Cheng \& de Jager 1990 for a discussion of theoretical models relevant to VHE gamma rays). Observational upper limits on the flux of radiated photons at the highest energies can put useful constraints on the various emission models. 


\section{Observational results}

\subsection{Crab nebula/pulsar and other EGRET pulsars}

A number of groups have now detected an excess 'DC' (i.e. unpulsed) flux from the Crab, whereas the results on the 'pulsed' signal, modulated with pulsar period, are apparently not consistent with each other (Table 1). The imaging technique is used by the Whipple, CANGAROO, HEGRA and Crimean groups. The Crab is the best studied object, but the highest energy emission detected so far is limited to $\sim 10 \mathrm{TeV}$. Observations higher than $\sim 10 \mathrm{TeV}$ have produced negative results with upper limits slightly above the spectrum extrapolated from lower energies (e.g. Borione et al. 1995). The VHE data do not yet cover a sufficiently wide range of energy with good enough accuracy and statistics, and the power indices of the integral spectrum of photon number reported to date range between -1.4 and -1.7 . However, when combined with the EGRET data, the global features of the Crab spectrum now allow us to argue about the identification of the origin of the target photons for the inverse Compton effect and the possible contribution of the bremsstrahlung process (Aharonian \& Atoyan 1995).

CANGAROO data show evidence of unpulsed gamma rays from the directions of the Vela pulsar and PSR B1706-44. As for Geminga, positive detections of a pulsed signal in early 1980s data have been claimed by the Durham and Tata groups. The more recent imaging Cerenkov result of the Whipple group gives an upper limit which is near the flux reported by the Tata group and is one order of magnitude smaller than the earlier result of the Durham group. For PSR $1951+32$, the Whipple upper limit on the DC flux is $5.4 \times 10^{-12} \mathrm{~cm}^{-2} \mathrm{~s}^{-1}$ (Weekes 1996). A preliminary analysis of CANGAROO data on PSR B1055-52 shows no evidence of detection, with an upper limit as small as $10^{-12} \mathrm{~cm}^{-2} \mathrm{~s}^{-1}$. No TeV observation has been reported on the newest EGRET pulsar PSR B0656+14 (Ramanamurthy et al. 1996).

\subsection{Millisecond/binary pulsars}

Table 2 lists VHE observations of short period pulsars. Evidence of pulsed emission was reported for PSR B1937+21 and for the binary pulsars, PSR B1953+29 and B1855+09, by the Durham group (Bowden et al. 1990; Chadwick et al. 1985). The Potchefstroom group saw evidence of emission from PSR B1855+09 (Brink et al. 1990), but an upper limit on the DC flux (irrespective of pulsed or unpulsed emission) obtained by Whipple group (Reynolds et al. 1993) is smaller than the flux of the positive detection reported. A hint of emission was reported also on PSR B1957+20, a binary pulsar with a $1.6 \mathrm{~ms}$ period, by the Potchefstroom group, but a later observation by the same group failed to reconfirm this (Brink et al. 1993). The upper limit of Durham group is smaller than the Potchefstroom flux. In a summary of Potchefstroom observations (Nel et al. 1992), the two pulsars PSR B1821-24 and B1923-13 are noted as having possibly shown transient VHE emission. The Tata groun (Bhat, et al. 1990) observed PSR B0355+54 with positive evidence which could not be confirmed in a later follow-up observation. The Gulmarg group reported evidence on DC flux of PSR B0355+54 (Senecha et al. 1995), which is, however, an order of magnitude larger than the upper limit to the DC flux obtained by the Whip- 
Table 1. Observations of the Crab, PSR B 1706-44, Vela and Geminga Group (reference) $\quad$ Flux $\left(\mathrm{cm}^{-2} \mathrm{~s}^{-1}\right) \quad \mathrm{E}_{\mathrm{th}}^{(*)}$ $========= \pm=$ Crab $===========$

- unpulsed -

Whipple (Lewis et al. 1993)

Crimea (Kalekin et al. 1995)

ASGAT (Goret et al. 1993)

$8.8 \times 10^{-12}(\mathrm{E} / \mathrm{TeV})^{-1.69} \quad 0.4$

$1.3 \times 10^{-11} \quad 1$

HEGRA (Konopelko et al. 1995)

$2.7 \times 10^{-11}$

0.6

Themistocle (Djannati-Atai et al. 1995)

CANGAROO (Tanimori et al. 1994)

$8 \times 10^{-12}(\mathrm{E} / \mathrm{TeV})^{-1.7} \quad 1$

- pulsed -

$2.3 \times 10^{-12}(\mathrm{E} / 3 \mathrm{TeV})^{-1.4} \quad 2.3$

Whipple (Gillanders et al. 1995)

$7.6 \times 10^{-13}$

7

Gulmarg (Sapru et al. 1996)

$<2.0 \times 10^{-13}$

0.25

Tata (Vishwanath et al. 1994)

$<2.5 \times 10^{-12}$

$(9.7 \pm 4.3) \times 10^{-12}$

4

Durham (Dowthwaite et al. 1984)

$(7.9 \pm 1.8) \times 10^{-12}$

$======= \pm===$ PSR B $1706-44==========$

- unpulsed -

CANGAROO (Kifune et al. 1995)

- pulsed -

Potchefstroom (Nel et al. 1993)

$8 \times 10^{-12}$

1

Potchefstroom (Nel et al. 1993)
$==========$ Vela pulsar $===========$
- unpulsed -

CANGAROO (in preparation)

$\sim 3 \times 10^{-12}$

- pulsed -

SAO/Sydney (Grindlay et al. 1975)

Tata(Bhat et al. 1987)

$<8.5 \times 10^{-11}$

0.3

Durham(Bowden et al. 1993a)

$\sim 1 \times 10^{-12}$

5

Potchefstroom(Nel et al. 1993)

$<5 \times 10^{-11}$

0.3

Adelaide(Edwards et al. 1994)

$<6 \times 10^{-12}$

2.3

$<7 \times 10^{-11}$

0.8

$==========$ Geminga $===========$

- unpulsed -

Whipple(Akerlof et al. 1993)

- pulsed -

Durham(Bowden et al. 1993b)

Tata(Vishwanath et al. 1993)

Whipple(Akerlof et al. 1993)

$<8.9 \times 10^{-12} \quad 0.5$

$3 \times 10^{-11}$

$(2.1 \pm 0.8) \times 10^{-11}$

1

$(4.4 \pm 3.5) \times 10^{-12}$

0.8

$<5 \times 10^{-12}$

1.7

0.5

$\left.{ }^{*}\right)$ : Threshold energy of detected gamma ray in $\mathrm{TeV}$. 
ple group (Reynolds et al. 1993). PSR B1259-63 (Johnston et al. 1996), in a highly eccentric binary orbit, presents an interesting site for pulsar wind experiments at varying distances between the pulsar and its massive companion. The CANGAROO group observed this object with a preliminary result on the DC flux at the $\sim 4 \sigma$ significance level, which encourages repeated observations. The binary pulsar PSR J0437-4715 of $5.8 \mathrm{~ms}$ period is interesting because of its distance of only $140 \mathrm{pc}$. An upper limit for the pulsed flux above $1 \mathrm{TeV}$ of $5 \times 10^{-11} \mathrm{~cm}^{-2} \mathrm{~s}^{-1}$ was reported by Dazeley et al. during this Colloquium.

Table 2. Observation list on millisecond and binary pulsars

\begin{tabular}{|c|c|c|c|c|}
\hline Nväninè & $P^{(*)}$ & Flux $\left(\operatorname{cini}^{-2} s^{-1}\right)$ & $E_{\text {th }}^{(* *)}$ & Gioup \\
\hline \multirow[t]{4}{*}{$0355+54$} & 156.4 & $7.9 \times 10^{-12}$ & 1.3 & Tata \\
\hline & & $<5.4 \times 10^{-13}$ & 3.4 & Tata \\
\hline & & $4.5 \times 10^{-11}$ & 4 ; DC? & Gulmarg \\
\hline & & $<4.0 \times 10^{-12}$ & $0.45 ; \mathrm{DC}$ & Whipple \\
\hline $0437-4715$ & 5.8 & $<5 \times 10^{-11}$ & 1 & BIGRAT \\
\hline $1259-63$ & 47.8 & a few times $10^{-12}$ & $3 ; \mathrm{DC}$ & CANGAROO \\
\hline $1821-24$ & 3.1 & transient & & Potchefstroom \\
\hline $1823-13$ & 101.5 & transient & & Potchefstroom \\
\hline \multirow[t]{3}{*}{$1855+09$} & 5.4 & $4 \times 10^{-10}$ & 0.4 & Durham \\
\hline & & $(2.3 \pm 2.5) \times 10^{-10}$ & 2 & Potchefstroom \\
\hline & & $<9.5 \times 10^{-12}$ & $0.6 ; \mathrm{DC}$ & Whipple \\
\hline $1937+21$ & 1.5 & $3 \times 10^{-11}$ & 1 & Durham \\
\hline $1953+29$ & 6.1 & $3 \times 10^{-11}$ & 2 & Durham \\
\hline $1957+20$ & 1.6 & $<2.8 \times 10^{-11}$ & 0.4 & Durham \\
\hline
\end{tabular}

Among the total 558 pulsars listed in Taylor et al. (1993) there are 48 pulsars with period shorter than $100 \mathrm{~ms}$, including 21 binary pulsars. Only Geminga and six radio pulsars have been found by EGRET to emit high energy gamma rays, with upper limits set for 40 other short period pulsars. The VHE survey with decent observation time is still limited to a small number of pulsars, and all the claims reported so far for detections of non-EGRET pulsars are of marginal significance. Efforts to achieve a more "complete" survey are necessary for arguing about emission models of VHE gamma rays from radio pulsars.

\section{Discussion}

\subsection{Brightness in the VHE gamma ray band}

In calculating the luminosity $\mathrm{L}_{\mathrm{VHE}}$ in the VHE gamma ray band from the reported flux, there are many factors which cause systematic errors; the threshold energy of the observations, the shape of energy spectrum to be integrated, contamination of the background (above which the gamma ray signal is detected), as well as the distance to pulsar. Some of these conditions depend on the individual observations, the details of which are not clearly known. Most of the positive 


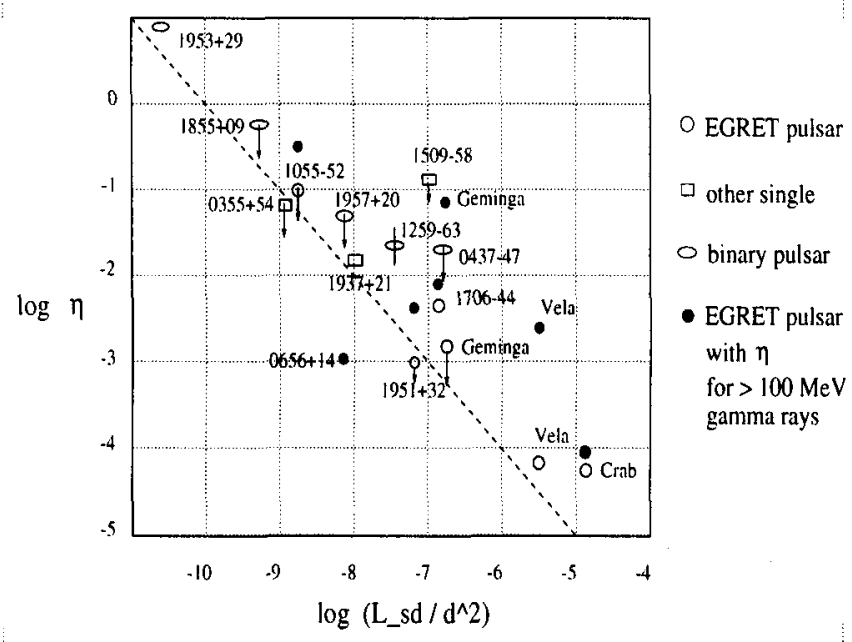

Figure 1. Scatter plot of brightness in VHE gamma rays and rotational energy loss of pulsars.

results on the millisecond pulsars are of claims for pulsed emission. In this case, there are uncertainties in the beaming factor of the emission angle and the duty cycle against the phase of pulsar rotation. We attempt in this paper to compare the VHE luminosity on a common base for different pulsars. The results plotted in Fig. 1 assume, for all observations, the same photon index of -2.5 and the same energy range of $500 \mathrm{GeV}$ to $10 \mathrm{TeV}$ despite the varying threshold energies from observation to observation. The distances listed in Taylor et al. (1993) are used. The solid angle of emission is taken to be $4 \pi$ and 1 steradian, respectively for unpulsed and pulsed emission. Some of the luminosities calculated here are considerably different from those given in the papers that reported the observation, which is not surprising in view of the existing uncertainties. In Fig. 1, the horizontal axis is $\log \left(\mathrm{L}_{\mathrm{sd}} / \mathrm{d}^{2}\right)$ in units of erg s $\mathrm{s}^{-1} \mathrm{~cm}^{-2}$, where $d$ is the distance to the earth, and the vertical axis is $\log \eta$, where $\eta$ is the ratio of gamma ray luminosity to spin down luminosity $\mathrm{L}_{\mathrm{sd}}$. The VHE luminosity is used for unfilled symbols $\left(\eta=\mathrm{L}_{\mathrm{VHE}} / \mathrm{L}_{\mathrm{sd}}\right)$, and the luminosity in $100 \mathrm{MeV}$ to $30 \mathrm{GeV}$ is used (instead of LVHE) for EGRET pulsars, which are shown with a black circle. Data of a same flux at the earth will appear in parallel with the dotted line which represents $\mathrm{L}_{\mathrm{VHE}} / \mathrm{d}^{2}=$ constant.

\subsection{Relation to other energy bands}

It appears that VHE emission from the EGRET pulsars is unpulsed: not modulated with pulsar period. This fact indicates that the VHE emission is not from the pulsar magnetosphere but from a remote site where pulsar wind collides with circumstellar matter. The VHE fluxes from the Crab, PSR B1706-44 and Vela correspond to luminosities of $\sim 2 \times 10^{34}, 10^{33} \sim 10^{34}$, and $10^{32} \sim 10^{33} \mathrm{erg} \mathrm{s}^{-1}$, respectively. For the latter two objects, the calculated value has an uncertainty of almost an order of magnitude depending on the power index and cutoff energy 
of the spectrum. With this in mind, by assuming that VHE gamma rays and Xray emission are from inverse Compton and synchrotron processes respectively, we can estimate the magnetic field by comparing the VHE and X-ray luminosities. The smaller X-ray luminosities than that of the Crab nebula yield weaker magnetic fields of $1 \sim 10 \mu \mathrm{G}$ for PSR B1706-44 and the Vela pulsar.

For the Vela pulsar, which is at a distance of only $\sim 500 \mathrm{pc}$, it is useful to know the geometrical structure of emission mechanism. The complex structure in the vicinity of Vela pulsar appears from X-ray data; the existence of a pointlike source at the pulsar, a $4^{\prime}$ nebula of diffuse emission, and hard X-ray emission extending from the pulsar to the radio feature Vela X (Harnden et al. 1985); soft $\mathrm{X}$-ray emission extending from the pulsar towards the south can be the result of non-thermal interactions by the pulsar wind (Markwardt \& Ögelman 1995), of which strong evidence would be given by gamma ray detection.

It is interesting to speculate on what type of pulsars would be bright at VHE gamma ray energies; pulsars which have accompanying synchrotron X-ray emission are likely VHE sources because of the existence of energetic electrons. Particle acceleration may take place at the shock generated by the pulsar wind that carries most of the rotational energy loss of the neutron star. The X-ray satellites ROSAT and ASCA are recently increasing the number of X-ray synchrotron nebula and/or pulsar wind sources. Also in this Colloquium, evidence of a pulsar wind was presented by N. Kawai from the ASCA group in a report of elongated emission bridging the synchrotron nebula of MSH 15-52 and the pulsar PSR B1509-58. The upper limit of VHE gamma rays reported for this object is not very stringent and a flux larger than the current sensitivity of imaging Cerenkov telescopes is estimated by du Plessis et al. (1995).

Less intense synchrotron X-rays are expected from lower magnetic fields (if the energy and number of progenitor electrons are kept constant) while more intense VHE gamma rays relative to the $\mathrm{X}$-ray intensity are expected. However, with low magnetic fields, the characteristic wavelength of synchrotron radiation can be in the UV to softer X-ray region, lower than the ROSAT and ASCA bands. In some pulsars, the energy spectrum of progenitor electrons can be steep, without continuing to high enough energy to produce detectable VHE emission. Thus, the existing X-ray and VHE gamma ray data are not good enough to allow detailed comparisons. The next generation of VHE telescopes is eagerly awaited, as these will be operating at energies lower than the current threshold of several hundred $\mathrm{GeV}$.

\subsection{Transient emission and Imaging technique of Čerenkov light}

The high $\eta$ values of some millisecond pulsars (Fig. 1) can suggest a very efficient mechanism of VHE emission and particle acceleration process. Similar results have been reported for on accretion-powered X-ray pulsars (Chadwick et al. 1990). Repeated observations are needed to resolve these puzzles. Difficulty in confirming the earlier results is due to the fact that most of the detections were obtained at a time of a burst-like increase of observed events which could enhance the significance of the time-correlated sigrial with the pulsar period. With imaging Cerenkov telescopes, however, a transient burst will appear as an increase of events with gamma ray-like features (i.e. in the imaging parameters such as the orientation angle $\alpha$ ) while cosmic ray background events can be 
monitored to ensure they stay constant with time. Imaging Ċerenkov technique observations of such transient phenomena will be very useful for examining the time-varying characteristics of such sources.

\section{Conclusions and Summary}

The ratio $\eta$ of the scatter plot in Fig. 1 appears at the $\sim 10^{-4}$ level for 'DC' emission of VHE gamma rays from the Crab and Vela pulsars, while of the order of $10^{-3}$ for PSR B1706-44. Pulsed VHE emission has not been detected at a. high confidence level for any of the EGRET pulsars, and the ratio is likely to be considerably smaller than the unpulsed case. There are interesting differences in the observational data among the three pulsars, Crab, Vela and PSR B1706-44. We need more data to compare more pulsars. The dissimilarities must be related to the different conditions of VHE emission in the pulsars.

The observed values and upper limits of the ratio $\eta$ are considerably larger for some pulsars than those of the Crab and Vela. A similar tendency of large $\eta$ and variations from pulsar to pulsar can be seen among the EGRET detections, as shown by the filled circles in Fig. 1. Pulsars with accompanying X-ray emission, such as PSR B1509-58, are worth extensive VHE observations. Binary pulsars such as PSR B1957+20 and B1259-63 can be bright in VHE gamma rays due to the interaction of the pulsar wind with a close companion star. The $500 \mathrm{pc}$ distance to the Vela pulsar means that the typical angular resolution of an imaging Cerenkov telescope, $0.1^{\circ}$, corresponds to about 1 pc at the source, which is not far from the size of pulsar wind shock of $0.1 \sim 1 \mathrm{pc}$ in the case of Crab (Kennel \& Coroniti 1984). If the pulsar wind causes non-thermal phenomena resulting in the emission of gamma rays in a close binary system like PSR B1259-63, the site of such a process is within $\sim 10^{13} \mathrm{~cm}$ from the pulsar, much less than the $\sim 0.1 \mathrm{pc}$ of the Crab. It is thus hoped that a more complete survey in VHE gamma rays of various types of pulsars will reveal interesting information on the behaviour of the pulsar wind such as the shock distance and strength of the magnetic field generated by pulsar wind.

The dotted line in Fig. 1 corresponds to a VHE gamma ray flux, $1.5 \times 10^{-12}$ $\mathrm{cm}^{-2} \mathrm{~s}^{-1}$, for photons $>1 \mathrm{TeV}$, which is roughly equal to the current sensitivity of imaging Cerenkov telescopes. The VHE data points in Fig. 1 are distributed almost along the dotted line, suggesting that the plotted data are influenced by detection biases and raising the hope that more sources may appear with continued efforts.

Acknowledgments. Thanks to P.G. Edwards for reading the manuscript and for comments and to the VHE colleagues who kindly informed the author of their latest results.

\section{References}

Aharonian, F.A. \& Atoyan, A.M., 1995, Astroparticle Phys. 3, 275

Akerlof, C.W. et al. 1993, A\&A, 274, L17

Bhat, P.N. et al. 1987, A\&A, 178, 242

Bhat, P.N. et al. 1990, A\&A, 236, L1 
Borione, A. et al. 1995, Proc. 24th Int. Cosmic Ray Conf., 2, 350

Bowden, C.G.G. et al. 1990, Nuclear Phys. B(Proc. Suppl.) 14A, 75

Bowden, C.G.G. et al. 1993a, Proc. 23rd Int. Cosmic Ray Conf., 1, 294

Bowden, C.G.G. et al. 1993b, J. Phys. G., 19, L29

Brink, C. et al. 1990, ApJ, 346, L37

Brink, C. et al. 1993, 23rd Proc. Int. Cosmic Ray Conf., 1, 334

Cawley, M.F. \& Weekes, T.C. 1995, Exp. Astron., 6, 742

Chadwick, P.M. et al. 1985, Nature, 310, 236

Chadwick, P.M., McComb, T.J.L., Turver, K.E. 1990, J. Phys. G., 16, 1773

Cheng, K.S. \& de Jager, O.C. 1990, Nuclear Phys. B(Proc. Suppl.), 14A, 28

Djannati-Atai, A. et al. 1995, Proc. 24th Int. Cosmic Ray Conf., 2, 315

Dowthwaite, T.C. et al. 1984, ApJ, 286, L35

du Plessis, I. et al. 1995, ApJ, 453, 746

Edwards, P.G. et al. 1994, A\&A, 291, 468

Gillanders, G.H. et al. 1995, Proc. 24th Int. Cosmic Ray Conf., 2, 323

Goret, P. et al. 1993, A\&A, 270, 401

Grindlay, J.E. et al, 1975, ApJ, 201, 82

Harnden, F.R. et al, 1985, ApJ, 299, 828

Johnston, S. et al, 1996, MNRAS, 279, 1026

Kalekin, O.G. et al. 1995, Astron. Lett., 21, 163

Kennel, C.F. \& Coroniti, F.V. 1984, ApJ, 283, 710

Kifune, T. et al. 1995, ApJ, 438, L91

Konopelko, A. et al. 1995, preprint MPIK-K-33-1995

Lewis, D.A. et al. 1993, Proc. 23rd Int. Cosmic Ray Conf., 1, 279

Markwardt, C.B. \& Ögelman, H. 1995, Nature, 375, 40

Nel, H.I. et al. 1992, ApJ, 398, 602

Nel, H.I. et al. 1993, ApJ, 418, 836

Ramanamurthy, P.V. et al. 1995, ApJ, 447, L109

Ramanamurthy, P.V. et al. 1996, ApJ, 458, 755

Reynolds, P.T. et al. 1993, ApJ, 404, 206

Sapru, M.L. et al. 1996, Bull. Astr. Soc. India, in press

Senecha, V.K. et al. 1995, A\&A, 302, 133

Tanimori, T. et al. 1994, ApJ, 429, L61

Taylor, J.H., Manchester, R.N., Lyne, A.G. 1993, Ap.JS, 88, 529

Vishwanath, P.R. et al. 1993, A\&A, 267, L5

Vishwanath, P.R. et al. 1994, Towards a Major Atmospheric Ćerenkov Detector

(Ed. T. Kifune, Academic Press, Tokyo), 65

Weekes, T.C. 1996, personal communication 